ORIGINAL RESEARCH

W.J. van Rooij

\section{Endovascular Treatment of Cavernous Sinus Aneurysms}

BACKGROUND AND PURPOSE: Aneurysms of the cavernous segment of the internal carotid artery generally exhibit a benign clinical course, with mass effect on cranial nerves. Rupture generally leads to carotid cavernous fistula and, rarely, to subarachnoid hemorrhage. In this study we report results of treatment in 85 patients with 86 cavernous sinus aneurysms.

MATERIALS AND METHODS: In a 15-year period, 85 patients with 86 cavernous sinus aneurysms were treated. There were 77 women (91\%) and 8 men, with a mean age of 55.5 years (range $26-78$ years). Presentation was cranial neuropathy in 56 , carotid cavernous fistula in 8 , and subarachnoid hemorrhage in 1 patient. Twenty-one aneurysms were asymptomatic. Treatment was selective coiling in 31 aneurysms and carotid artery occlusion in 55 aneurysms, 5 after bypass surgery.

RESULTS: All 8 cavernous sinus fistulas were closed with coils. There were no complications of coiling and 1 patient had a permanent neurologic complication after carotid artery occlusion (morbidity 1.2\%; $95 \%$ confidence interval, 0.01 to $6.9 \%$ ). Clinical and MR imaging follow-up ranged from 3 months to 12 years. In 52 of 56 (93\%) patients presenting with symptoms of mass effect, symptoms either were cured $(n=23)$ or improved $(n=29)$. All aneurysms were thrombosed after carotid artery occlusion and at latest MR imaging, 34 of 50 aneurysms (68\%) were substantially decreased in size or completely obliterated.

CONCLUSIONS: In this series, for patients with cavernous sinus aneurysms, a treatment strategy including selective coiling and carotid artery occlusion was safe and effective. Most symptomatic patients (93\%) were improved or cured, and most aneurysms (68\%) shrank on follow-up.
A neurysms of the cavernous segment of the internal carotid artery form a distinct entity. These aneurysms can produce symptoms through mass effect of the aneurysm on adjacent cranial nerves or through rupture, which leads to carotid cavernous fistula or, occasionally, to subarachnoid hemorrhage, if the aneurysm extends through the dura. Mass effect on cranial nerves III-VI may cause retro-orbital pain, diplopia in primary or secondary gaze, ophthalmoparesis, ophthalmoplegia, trigeminal neuropathy, or a combination of these. Symptom onset may be gradual or acute, the latter with aneurysm wall dissection resulting in sudden aneurysm growth. The natural history of cavernous sinus aneurysms is not fully understood. The risk of subarachnoid hemorrhage is low, with an estimated risk of $0.2-0.4 \%$ per patient year. The course of cranial nerve dysfunction by mass effect seems variable, often with progression of symptoms and also spontaneous improvement in a substantial proportion of patients. ${ }^{1-6}$ In view of the relatively benign course of cavernous sinus aneurysms, any treatment should have a low complication risk and should have a reasonable chance for clinical improvement in symptomatic patients. Treatment alternatives traditionally consist of selective aneurysm coiling or internal carotid artery occlusion, with or without preceding bypass surgery. This study reports the results of treatment of 86 cavernous sinus aneurysms during a 15 -year period in a single institution.

Received March 22, 2011; accepted after revision June 1

From the Department of Radiology, St. Elisabeth Ziekenhuis, Tilburg, the Netherlands.

Please address correspondence to Willem Jan van Rooij, Department of Radiology, St. Elisabeth Ziekenhuis, Hilvarenbeekseweg 60, 5022GC Tilburg, the Netherlands; e-mail: radiol@eztilburg.nl

http://dx.doi.org/10.3174/ajnr.A2759

\section{Materials and Methods}

\section{General Indications for Treatment}

Patients with cavernous carotid aneurysms were discussed in a joint meeting with neurologists, neurosurgeons, and neuroradiologists. Treatment was tailored to the individual patient, accounting for clinical presentation, patient age, comorbidity, and patient wishes. Patients with ruptured cavernous sinus aneurysms (carotid cavernous fistula or subarachnoid hemorrhage) were offered endovascular treatment. Patients with cavernous sinus aneurysms, symptomatic by mass effect on cranial nerves III-VI (retro-orbital pain, ophthalmoplegia, ophthalmoparesis, trigeminal neuropathy), were also offered treatment. In patients with asymptomatic cavernous sinus aneurysms that were either incidentally discovered or additional to another symptomatic aneurysm, treatment offer was dependent on several patient and aneurysm characteristics, such as patient age, comorbidity, aneurysm size, and aneurysm neck size. For small aneurysms with a small or intermediate neck size, coiling was the preferred treatment. For large and giant aneurysms, carotid artery occlusion was considered first. If test occlusion indicated nontolerance, either conservative therapy or bypass surgery was offered, dependent on several factors, such as clinical presentation, patient age, comorbidity, and patient wishes.

\section{Endovascular Treatment Modalities}

In general, coiling was the preferred treatment for small aneurysms and for all ruptured cavernous sinus aneurysms. For large and giant aneurysms, ICA occlusion was the preferred treatment. When ICA occlusion was not tolerated, alternatives were selective coiling, bypass surgery preceding ICA occlusion, or conservative therapy.

\section{Coiling Procedure}

Coiling was performed under general anesthesia on a biplane angiographic unit (Philips Integris or Allura Neuro; Philips Healthcare, Best, the Netherlands). Wide-neck aneurysms were coiled with 
balloon assistance or, in the last years of the study period, after stent placement. Flow diverters were not used. In some patients, a test occlusion was performed during the coiling procedure as a bailout for complications or for possible future counseling. Heparin was intraarterially administered during coiling and continued subcutaneously for 48 hours. Patients with stent placement were preloaded with clopidogrel that was continued for 3-6 months.

\section{Internal Carotid Artery Occlusion}

ICA occlusion was performed according to a previously described protocol. ${ }^{7}$ In short, during balloon test occlusion in the awake patient, angiography was performed of the contralateral carotid artery and/or vertebral artery to assess collateral flow via the anterior and posterior communicating arteries. Apart from clinical tolerance, synchronous opacification of the veins in the territories of the examined and occluded vessels was considered indicative of tolerance to permanent occlusion. After passing the test, the ICA was occluded with detachable balloons or, in a short period where balloons were unavailable, with coils. After permanent ICA occlusion, the patient was monitored in a medium care unit for 24 hours.

\section{Bypass Surgery}

Bypass surgery was considered in symptomatic patients with wideneck large or giant aneurysms who could not tolerate carotid artery occlusion. After constructing a high-flow bypass, according to a previously described protocol, ${ }^{8,9}$ the internal carotid artery was occluded with detachable balloons.

\section{Follow-Up}

After ICA occlusion, clinical follow-up and MR imaging was scheduled after 3 months and, in many patients, at various intervals thereafter. Some patients were the subject of long-term MR imaging follow-up studies. ${ }^{10,11}$ After coiling, angiographic follow-up was scheduled after 6 months and, depending on results, extended angiographic or MR imaging follow-up was scheduled at various intervals. In the second half of the study period, angiographic follow-up was largely replaced by MRA at 3T.

\section{Patients}

Between January 1995 and October 2010, in 104 patients with 105 cavernous sinus aneurysms, treatment was considered. Of these, 19 patients with aneurysms not suitable for coiling could not tolerate ICA occlusion and opted for conservative treatment rather than bypass surgery. These 19 patients were censored from this study. The remaining 85 patients with 86 cavernous sinus aneurysms were actually treated at our institution and these patients form the subject of the study. One patient had bilateral cavernous sinus aneurysms that were both coiled. Patient and aneurysm characteristics are summarized in the Table. Of 86 aneurysms, 31 in 30 patients (36\%) were treated with selective coiling and 55 (64\%) were treated with internal carotid artery occlusion, 5 after previous bypass surgery. Two patients were treated with coiling first and were later treated with ICA occlusion for the same aneurysm. These patients are evaluated as ICA occlusion only. Mean patient age was 55.5 years, and 77 of 85 (90.6\%) patients were female. Three patients had an additional large or giant opposite carotid-ophthalmic aneurysm that was coiled. In 8 patients who presented with a carotid cavernous fistula, the fistula was closed with coils; in 6 patients, with sparing of the ICA; and in 3 patients, with concomitant occlusion of the ICA (Fig 1). Of 31 coiled aneurysms, 8 were coiled with balloon assistance and 2 after stent placement (En-

\begin{tabular}{|c|c|c|c|c|}
\hline \multicolumn{5}{|c|}{$\begin{array}{l}\text { Patient, aneurysm, and treatment characteristics of } 85 \text { patients with } \\
86 \text { treated cavernous sinus aneurysms }\end{array}$} \\
\hline & \multicolumn{3}{|c|}{ ICA } & \multirow[b]{2}{*}{ Total } \\
\hline & Coiling & Occlusion & Bypass & \\
\hline Patients & 30 & 50 & 5 & 85 \\
\hline Aneurysms & 31 & 50 & 5 & 86 \\
\hline Mean patient age (years) & 53.3 & 57.6 & 48.2 & 55.5 \\
\hline Median age, range & $56,27-72$ & $58,26-79$ & $47,34-56$ & $56.5,26-79$ \\
\hline Male gender & $4(13 \%)$ & $4(8 \%)$ & $0(0 \%)$ & $8(9.4 \%)$ \\
\hline Mean aneurysm size (mm) & 10 & 21 & 27 & \\
\hline Median size, range & $6,5-35$ & $18,12-45$ & $25,20-40$ & \\
\hline \multicolumn{5}{|l|}{ Clinical presentation } \\
\hline Cranial nerve dysfunction & 6 & 45 & 5 & $56(65 \%)$ \\
\hline Carotid cavernous fistula & 5 & 3 & 0 & $8(9 \%)$ \\
\hline Subarachnoid hemorrhage & 1 & 0 & 0 & $1(1 \%)$ \\
\hline Incidental finding & 4 & 2 & 0 & $6(7 \%)$ \\
\hline $\begin{array}{l}\text { Additional to another } \\
\text { aneurysm }\end{array}$ & 15 & 0 & 0 & $15(17 \%)$ \\
\hline
\end{tabular}

terprise stent; Cordis, Miami Lakes, Florida). Of 56 aneurysms that presented with cranial nerve dysfunction, 50 (89\%) were treated with ICA occlusion. On the other hand, of 21 asymptomatic aneurysms, 19 (91\%) were treated with selective coiling.

\section{Results}

\section{Internal Carotid Artery Occlusion}

After carotid artery occlusion, MR imaging follow-up showed complete aneurysm thrombosis in all. There were no complications from bypass surgery in 5 patients. Of 50 patients with ICA occlusion without previous bypass, 2 developed small hypoperfusion infarctions in the territory of the occluded ICA, with temporal 1-sided weakness. One 79-year-old patient developed a large retroperitoneal hematoma that had to be surgically evacuated. The extensive blood loss caused a hypoperfusion infarction in the territory of the occluded ICA. Altogether, there was 1 patient with a permanent neurologic deficit as an direct result of the ICA occlusion, and there were no deaths (morbidity, 2\% [ 1 of 50]; 95\% confidence interval, 0.01 to $11.5 \%$; mortality, $0 \%$; $95 \%$ confidence interval, 0 to $8.5 \%)$.

\section{Coiling}

There were no complications in coiling of 31 aneurysms ( $0 \%$; $95 \%$ confidence interval, 0 to $13.1 \%$ ). Aneurysm occlusion after coiling was complete in 10 , near complete in 17 , and incomplete in 4 aneurysms. All 8 carotid cavernous fistulas were closed.

\section{Clinical and Imaging Follow-Up}

In the 30 patients with 31 coiled cavernous sinus aneurysms, the mean clinical follow-up was 42 months (median 24, range 6-146 months) and the mean imaging follow-up was 38 months (median 18, range 6-146 months). During follow-up, 6 aneurysms were additionally coiled. Of 6 aneurysms that presented with mass effect, symptoms were cured in 2 and improved in 4 . Transient increase in symptoms after treatment did not occur.

In 55 patients with 55 aneurysms that were treated with ICA occlusion, mean clinical and imaging follow-up was 12 months (median 4, range 3-121 months). Of 50 patients who presented with cranial nerve dysfunction, 21 were cured 


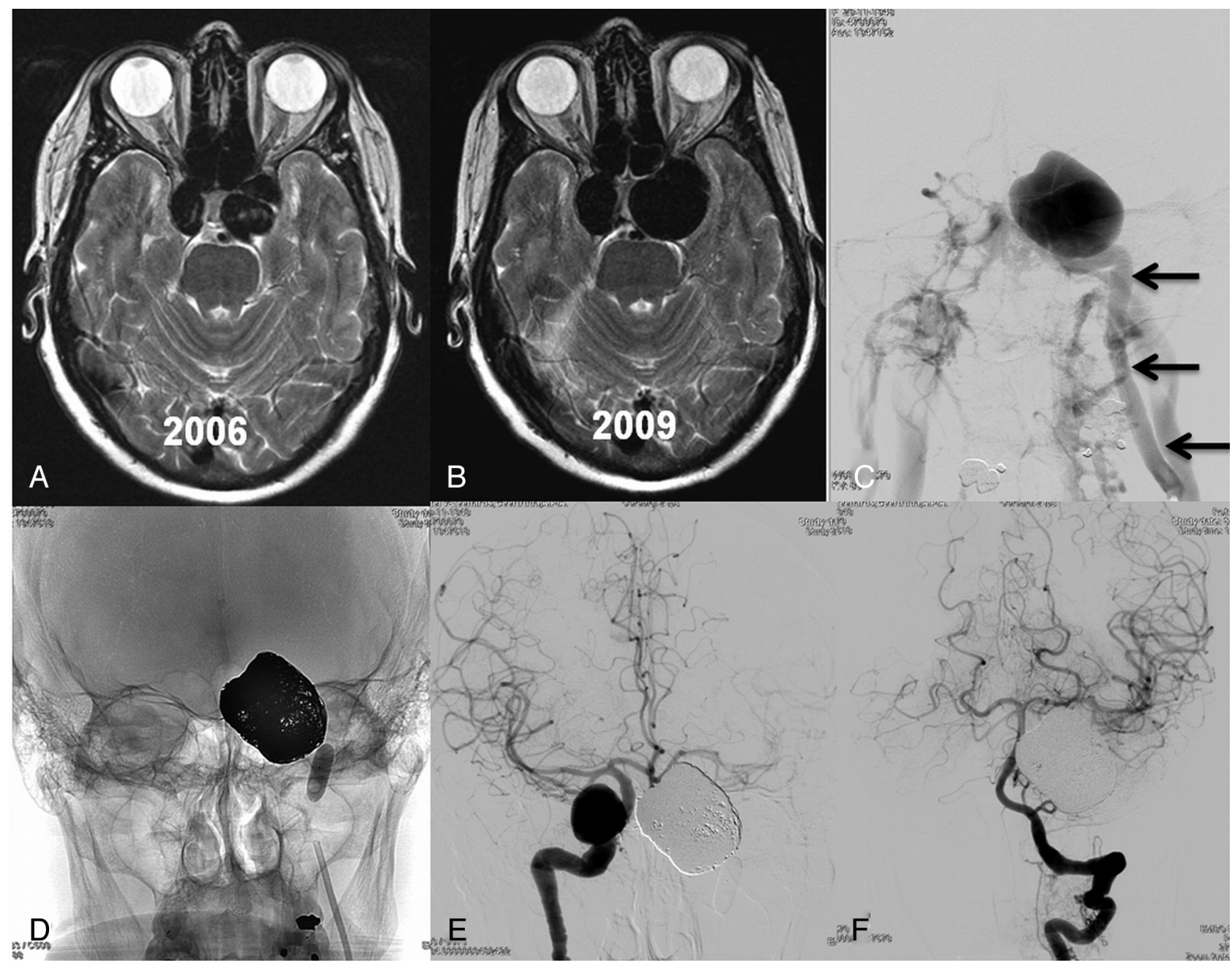

Fig 1. 62-year-old woman with incidentally discovered bilateral cavernous sinus aneurysms. $A$, MR imaging for headaches in 2006 shows bilateral cavernous sinus aneurysms. Treatment was not recommended. $B$, MR imaging 3 years later demonstrates growth of both aneurysms. The patient was asymptomatic. $C$, In 2010 , the left aneurysm ruptured causing a carotid cavernous fistula with proptosis and ophthalmoplegia. Left carotid angiogram in frontal view shows early filling of both cavernous sinus and many skull base and neck veins. There was no filling of intracranial vessels. Arrows indicate internal carotid artery. $D$, The aneurysm was coiled; the carotid artery was also occluded with coils and sealed with a balloon. Clinical symptoms were cured within 2 weeks. $E$ and $F$, Frontal views of right internal carotid artery $(E)$ and left vertebral artery $(F)$ after left internal carotid artery occlusion demonstrate collateral flow to the left hemisphere via the circle of Willis.

(42\%), 25 improved (50\%), 3 were unchanged (6\%), and 1 patient $(2 \%)$ was hemiplegic after a complication. At latest MR imaging, 34 of 50 aneurysms (68\%) were substantially decreased in size $(50 \%-75 \%$ decrease in size) or completely obliterated (Fig 2).

\section{Overall Results}

In 85 patients with 86 treated aneurysms, 1 procedural complication, with permanent neurologic deficit, occurred $(1.2 \%$; $95 \%$ confidence interval, 0.01 to $6.9 \%$ ). Of 56 patients presenting with symptoms of mass effect, symptoms were either cured $(n=23)$ or improved $(n=29)$ in 52 patients $(93 \%)$.

\section{Discussion}

In this study, we report the results of a management strategy in the treatment of cavernous sinus aneurysms of a single center over a long period of time. In view of the relatively benign clinical course of these aneurysms, we tried to tailor the treatment strategy to the individual patient by balancing the risk of treatment against the possible clinical benefit. A drawback of our study is that our population is treatment-based, and we did not record patients with cavernous sinus aneurysms who were referred with imaging studies from other centers and were not treated with endovascular or surgical techniques (apart from the patients in whom carotid test occlusion indicated nontolerance and who opted for conservative treatment). In particular, we do not know how many patients with symptomatic cavernous sinus aneurysms were not treated. The clinical course in these patients may be variable, and indication for invasive treatment might have changed with changing symptoms.

Our results confirmed the striking preponderance of cavernous sinus aneurysms in women. ${ }^{1-5}$ We found that both selective coiling and endovascular carotid artery occlusion for cavernous sinus aneurysms is safe. The test occlusion protocol for carotid artery occlusion, consisting of clinical observation and synchronous venous filling on angiography, has proved to be reliable in predicting acute and delayed cerebral ischemia after parent artery occlusion. After carotid artery occlusion, all aneurysms thrombosed and most decreased in size or were 

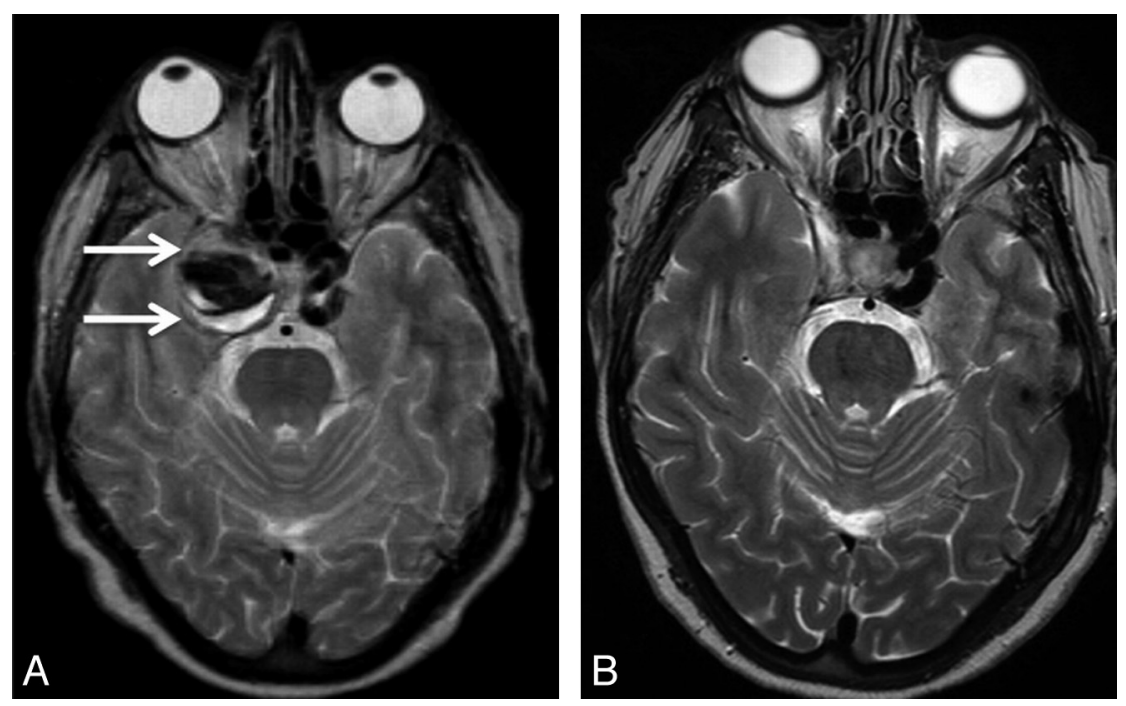

Fig 2. 54-year-old woman with ophthalmoplegia by mass effect of a giant right cavernous sinus aneurysm, treated with carotid artery occlusion. $A, \mathrm{MR}$ imaging demonstrates the giant cavernous sinus aneurysm (arrows). B, On follow-up MR imaging 18 months later, the aneurysm is completely obliterated; ophthalmoplegia was improved to isolated abducens palsy. There was no diplopia in primary gaze.

completely obliterated, generally in the first year after treatment. Most symptomatic patients showed clinical cure or improvement of cranial nerve dysfunction induced by large or giant unruptured cavernous sinus aneurysms, both after ICA occlusion and after selective coiling, and generally in the first year after treatment. In only 7 of 48 aneurysms, symptoms of cranial nerve deficits remained unchanged after therapy and aggravation of symptoms did not occur. The recovery process of cranial nerves may be dependent on several factors, such as duration of symptoms before treatment, acute or gradual onset of symptoms, degree of cranial nerve dysfunction (complete versus partial), and presence of microvascular risk factors such as hypertension, diabetes, and advanced age. Our results are in line with other studies. ${ }^{12,13}$

In patients presenting with a carotid cavernous fistula following rupture of a cavernous sinus aneurysm, the treatment strategy is different from unruptured aneurysms. Simple balloon occlusion of the carotid artery proximal to the ruptured aneurysm may induce retrograde flow via the circle of Willis into the fistula. Trapping the fistula with 2 balloons in the carotid artery proximal and distal to the aneurysm is technically difficult, especially passing the fistula with a balloon. Closing the fistula by coiling of the aneurysm, if necessary, in combination with coil occlusion of the carotid artery, proved to be simple, effective, and safe.

Although 5 patients in this study had uncomplicated highflow bypass surgery by using the Excimer laser-assisted anastomosis technique preceding carotid artery occlusion, we were reluctant in offering this therapy because of its rather high chance of complications. ${ }^{9}$ Recent sophisticated endovascular techniques that spare the carotid artery may be an alternative to bypass surgery: New flow-diverting stents are designed to occlude the aneurysm while reconstructing the carotid artery. ${ }^{14}$ Although the first clinical results are very encouraging, the safety and effectiveness of these devices is not yet known. In addition, the required prolonged antiplatelet medication is a disadvantage. Future studies have to define the role of these flowdiverting stents in the treatment of cavernous sinus aneurysms.

\section{Conclusions}

Cavernous sinus aneurysms can be treated, with few complications, with coiling or carotid artery occlusion. Symptoms of mass effect improve or are cured in most patients. In patients who cannot tolerate carotid artery occlusion, the risk of treatment of bypass surgery or sophisticated endovascular coiling or stent techniques sparing the carotid artery should be carefully balanced against the often-benign clinical course of cranial neuropathy.

\section{References}

1. Linskey ME, Sekhar LN, Hirsch WL, et al. Aneurysms of the intracavernous carotid artery: natural history and indications for treatment. Neurosurgery 1990;26:933-37

2. Kupersmith MJ, Hurst R, Berenstein A, et al. The benign course of cavernous carotid artery aneurysms. J Neurosurg 1992;77:690-93

3. Kupersmith MJ, Stiebel-Kalish H, Huna-Baron R, et al. Cavernous carotid aneurysms rarely cause subarachnoid hemorrhage or major neurologic morbidity. J Stroke Cerebrovasc Dis 2002;11:9-14

4. Stiebel-Kalish H, Kalish Y, Bar-On RH, et al. Presentation, natural history, and management of carotid cavernous aneurysms. Neurosurgery 2005;57:850-57

5. Goldenberg-Cohen N, Curry C, Miller NR, et al. Long term visual and neurological prognosis in patients with treated and untreated cavernous sinus aneurysms. J Neurol Neurosurg Psychiatry 2004;75:863-67

6. Van der Schaaf IC, Brilstra EH, Buskens E, et al. Endovascular treatment of aneurysms in the cavernous sinus: a systematic review on balloon occlusion of the parent vessel and embolization with coils. Stroke 2002;33:313-18

7. van Rooij WJ, Sluzewski M, Slob MJ, et al. Predictive value of angiographic testing for tolerance to therapeutic occlusion of the carotid artery. AJNR Am J Neuroradiol 2005;26:175-78

8. van Doormaal TP, van der Zwan A, Verweij BH, et al. Treatment of giant and large internal carotid artery aneurysms with a high-flow replacement bypass using the Excimer laser-assisted nonocclusive anastomosis technique. Neurosurgery 62 (6 Suppl 3):1411-18, 2008

9. Brilstra EH, Rinkel GJ, Klijn CJ, et al. Excimer laser-assisted bypass in aneurysm treatment: short-term outcomes. J Neurosurg 2002;97:1029-35

10. de Gast AN, Sprengers ME, van Rooij WJ, et al. Midterm clinical and magnetic resonance imaging follow-up of large and giant carotid artery aneurysms after therapeutic carotid artery occlusion. Neurosurgery 2007;60:1025-29

11. de Gast AN, Sprengers ME, van Rooij WJ, et al. Long term 3T-MRA follow-up after therapeutic occlusion of the internal carotid artery to detect possible de novo aneurysm formation. AJNR Am J Neuroradiol 2007;28:508-10

12. van Rooij WJ, Sluzewski M. Unruptured large and giant carotid artery aneurysms presenting with cranial nerve palsy: comparison of clinical recovery after selective aneurysm coiling and therapeutic carotid artery occlusion. AJNR Am J Neuroradiol 2008;29:997-1002

13. Mansour N, Kamel MH, Kelleher M, et al. Resolution of cranial nerve paresis after endovascular management of cerebral aneurysms. Surg Neurol 2007;68:500-04

14. Nelson PK, Lylyk P, Szikora I, et al. The Pipeline embolization device for the intracranial treatment of aneurysms trial. AJNR Am J Neuroradiol 2011;32:34-40 